\title{
Neumonía por Pneumocystis jirovecii en pacientes sin infección por VIH
}

\section{Pneumocystis jirovecii pneumonia in non-HIV-infected patients}

Jaime Andrés Alvarado, MD(1); Jacqueline Pavía, MD(1); Mónica Caicedo, $\mathrm{MD}^{(1)}$; María Ximena Chacón, $\mathrm{MD}^{(2)}$; Bibiana Pinzón, $\mathrm{MD}^{(3)}$; Margarita Baldión, $\mathrm{MD}^{(4)}$

\section{RESUMEN}

La neumonía por Pneumocystis jirovecii es una entidad relativamente infrecuente por fuera del contexto de la infección por virus de inmunodeficiencia humana $(\mathrm{VIH})$. No obstante, se espera un aumento en su incidencia en pacientes con otras causas de compromiso secundario de la inmunidad sobre todo teniendo en cuenta la proliferación de procedimientos relacionados con trasplante de órganos y la disponibilidad creciente de medicamentos inmunosupresores. La presentación clínica y radiológica es diferente a la reportada usualmente en pacientes con SIDA. La evolución es más agresiva y su pronóstico más oscuro.

Palabras clave: VIH, Pneumocystis jirovecii, neumonía, inmunosupresión.

\section{ABSTRACT}

Pneumocystis jirovecii pneumonia is relatively uncommon outside the context of HIV infection. However, it is expected that its incidence will increase in patients with other causes of secondary immune impairment, especially considering the proliferation of organ transplant procedures and the growing availability of immunosuppressant drugs. Clinical presentation and radiologic findings are usually different from those reported in patients with AIDS. The evolution is more aggressive and the prognosis more somber.

Keywords: HIV, Pneumocystis jirovecii, pneumonia, immunosuppression.

\section{INTRODUCCIÓN}

La neumonía por Pneumocystis jirovecii ha sido considerada históricamente como una de las infecciones oportunistas más frecuentes en pacientes con síndrome de inmunodeficiencia adquirida (SIDA), aunque con el advenimiento de la terapia antirretroviral altamente efectiva (HAART) y el uso de profilaxis, su incidencia viene en descenso, caso contrario a lo que ocurre en los pacientes con inmunosupresión de otras etiologías, en especial las inducidas por medicamentos en el contexto de trasplante, malignidad y enfermedad autoinmune. En este artículo se revisa una serie de cuatro casos de neumonía por Pneumocystis jirovecii en pacientes sin infección por $\mathrm{VIH}$ : estado postrasplante hepático por cirrosis biliar primaria, macroglobulinemia de Waldenstrom, cirrosis biliar primaria asociada a anemia hemolítica autoinmune y estado postrasplante hepático por cirrosis por hepatitis C.

\footnotetext{
(1) Neumólogo, Fundación Santa Fe de Bogotá, Colombia.

(2) Residente de Medicina Interna, Universidad El Bosque, Fundación Santa Fe de Bogotá, Colombia.

(3) Radióloga, Fundación Santa Fe de Bogotá, Colombia.

(4) Patóloga, Fundación Santa Fe de Bogotá , Colombia.
}

Correspondencia: Jaime Andrés Alvarado. Correo electrónico: jaimealvarado@gmail.com

Recibido: 24-02-2013. Aceptado: 13-03-2013. 


\section{RESULTADOS}

Se encontraron cuatro pacientes con diagnóstico de neumonía por Pneumocystis jirovecii tratados en la Fundación Santa Fe, en los últimos dos años (tabla 1). Tres de los cuatro pacientes cursaban con patología hepática, dos ellos en estado postrasplante de duración mayor a un año en manejo inmunosupresor. El caso restante correspondió a un diagnóstico de macroglobulinemia de Waldenstrom. El uso de dosis altas de esteroides previas al diagnóstico fue el común denominador en los cuatro casos. Dos de los pacientes tenían manejo concomitante con otros inmunosupresores. Los síntomas más frecuentes fueron tos, fiebre y disnea en su orden. Los hallazgos más prevalentes fueron desaturación y cambios en la auscultación pulmonar. Todos presentaron cambios patológicos en la radiografía de tórax y en la tomografía de tórax de alta resolución (TACAR). La hipoxemia con diferencia alvéolo-arterial (DA-a) aumentada estuvo presente en tres de los cuatro casos. El rendimiento diagnóstico del lavado broncoalveolar fue excelente con una buena correlación con las biopsias transbronquiales. No se realizaron serologías ni análisis de biología molecular. No se requirió biopsia pulmonar por método quirúrgico. Dos de los casos presentaban coinfecciones significativas: colitis por citomegalovirus y tuberculosis pulmonar. La mortalidad fue del $25 \%$, la cual correspondió al paciente más sintomático y con presentación radiológica y clínica más severa desde el inicio. Los tres pacientes restantes transcurrieron con cuadros de leves a moderados con curso benigno y buena respuesta terapéutica, asociada a recuperación completa sin secuelas clínicas ni paraclínicas. Un paciente desarrolló erupción cutánea como manifestación de alergia a las sulfas por lo cual requirió medicamentos de segunda línea. La mitad de los pacientes se trató con esteroide sistémico. Los dos pacientes postrasplante recibieron por protocolo profilaxis con trimetroprimsulfametoxazol (TMP-SMX) durante los primeros seis meses posinjerto.

\section{DISCUSIÓN}

La neumonía por Pneumocystis jirovecii ha sido reconocida ampliamente como infección oportunista en el contexto de la infección por VIH. De hecho, el Pneumocystis jirovecii ganó notoriedad en la comunidad científica como marcador de la enfermedad durante los primeros años de la epidemia de SIDA en los ochenta. No obstante, su descubrimiento como ente causal de enfermedad pulmonar en humanos data de 1940 (1), cuando fue descrito como el agente etiológico de alguno tipos raros y fatales de neumonía en grupos de niños prematuros, en desnutridos y posteriormente en pacientes con neoplasias hematológicas. Con un ciclo de vida complejo y una amplia distribución en el ambiente, el Pneumocystis jirovecii actualmente está clasificado dentro del filo de los hongos, no sin antes haber tenido lugar una profunda controversia por sus múltiples similitudes con los parásitos. Existen excelentes revisiones sobre la microbiología y la fisiopatología de este patógeno por lo que los autores consideran que estos tópicos exceden el objeto de esta revisión y remiten a estas publicaciones $(1,2)$.

En el caso del paciente inmunosuprimido no $\mathrm{VIH}$, la neumonía por Pneumocystis jirovecii se convierte en un desafío para el clínico teniendo en cuenta las diferencias marcadas en la presentación clínica y en el pronóstico. Por ello se debe combinar el interrogatorio enfocado en los factores de riesgo con una alta sospecha diagnóstica basada en hallazgos paraclínicos. Con el auge del uso de terapia inmunosupresora, en diferentes contextos como medicina de trasplantes, oncología, hematología y reumatología, la incidencia de Pneumocystis jirovecii en el paciente no VIH ha ido en aumento (3). El cuadro neumónico puede darse como el resultado de una infección de novo (adquirida en la comunidad o nosocomial) o por reactivación de una infección latente (colonización). Adicionalmente, se debe tener en cuenta que el riesgo de desarrollar neumonía por Pneumocystis jirovecii es directamente proporcional a la intensidad y la duración del estado de inmunosupresión (4).

Se han descrito casos de infección por Pneumocystis jirovecii en malignidades hematológicas, tumores sólidos, trasplante de órgano sólido y de células madre hematopoyéticas y en las enfermedades del tejido conectivo que se encuentran bajo la terapia inmunosupresora (5). El desarrollo de la infección está relacionado con la inmunosupresión, por sí misma, de la enfermedad aunado al uso de agentes inmunosupresores como corticosteroides, análogos de las purinas, inhibidores de calcineurina y terapia biológica (6). El hecho de que en enfermedades y tratamientos diversos con diferente efecto sobre la respuesta inmune se hayan reportado casos de infección por este microorganismo, hace notorio el que la interacción entre el sistema inmune y el Pneumocystis jirovecii no solo está determinada por la deficiencia de células CD4, condición obvia en el paciente con $\mathrm{VIH}$, sino que en ella también desempeña un papel crucial el reconocimiento del Pneumocystis jirovecii, una respuesta antígeno anticuerpo exagerada, la producción de factor de necrosis tumoral con reclutamiento 
Tabla 1. Revisión de casos.

\begin{tabular}{|c|c|c|c|c|}
\hline Paciente & 1 & 2 & 3 & 4 \\
\hline Sexo & M & $\mathrm{F}$ & $\mathrm{F}$ & $M$ \\
\hline Edad & 45 años & 66 años & 55 años & 39 años \\
\hline Diagnóstico & $\begin{array}{l}\text { Por trasplante } \\
\text { hepático por cirrosis } \\
\text { biliar primaria }\end{array}$ & $\begin{array}{l}\text { Macroglobulinemia } \\
\text { de Waldenstrom }\end{array}$ & $\begin{array}{l}\text { Cirrosis biliar } \\
\text { primaria }\end{array}$ & $\begin{array}{l}\text { Pop trasplante } \\
\text { hepático por } \\
\text { hepatitis C y } \\
\text { hepatocarcinoma }\end{array}$ \\
\hline Comorbilidades & Colitis ulcerativa & No & $\begin{array}{l}\text { Púrpura } \\
\text { trombocitopénica } \\
\text { autoinmune }\end{array}$ & DM 2 \\
\hline Uso previo de esteroide & $\mathrm{Si}$ & $\mathrm{Si}$ & $\mathrm{Si}$ & $\mathrm{Si}$ \\
\hline Otros inmunosupresores & Tacrolimus & No & No & $\begin{array}{l}\text { Tacrolimus, } \\
\text { micofenolato }\end{array}$ \\
\hline Tos & $\mathrm{Si}$ & $\mathrm{Si}$ & $\mathrm{Si}$ & $\mathrm{Si}$ \\
\hline Fiebre & $\mathrm{Si}$ & No & $\mathrm{Si}$ & $\mathrm{Si}$ \\
\hline Disnea (MRC) al inicio & 1 & 1 & 4 & 3 \\
\hline $\begin{array}{l}\text { Cambios en la } \\
\text { auscultación pulmonar }\end{array}$ & No & Estertores basales & Estertores basales & Estertores basales \\
\hline $\begin{array}{l}\text { Signos de dificultad } \\
\text { respiratoria }\end{array}$ & No & No & $\mathrm{Si}$ & No \\
\hline $\begin{array}{l}\text { Hallazgos en radiografía } \\
\text { de tórax }\end{array}$ & $\begin{array}{l}\text { Opacidades } \\
\text { reticulares }\end{array}$ & $\begin{array}{l}\text { Opacidades } \\
\text { reticulonodulares }\end{array}$ & $\begin{array}{l}\text { Opacidades } \\
\text { reticulares }\end{array}$ & $\begin{array}{l}\text { Opacidades } \\
\text { reticulonodulares }\end{array}$ \\
\hline Hallazgos en TACAR & $\begin{array}{l}\text { Opacidades en vidrio } \\
\text { esmerilado }\end{array}$ & $\begin{array}{l}\text { Opacidades en } \\
\text { vidrio esmerilado } \\
\text { con nódulos } \\
\text { centrolobulillares }\end{array}$ & $\begin{array}{l}\text { Opacidades en vidrio } \\
\text { esmerilado }\end{array}$ & $\begin{array}{l}\text { Micronódulos } \\
\text { aleatorios y árbol en } \\
\text { gemación }\end{array}$ \\
\hline Diferencial en BAL & Linfocitario & Normal & Neutrofílico & Linfocitario \\
\hline $\begin{array}{l}\text { Tinciones positivas en } \\
\text { BAL }\end{array}$ & Gomori & PAS & Gomori & Gomori y PAS \\
\hline $\begin{array}{l}\text { Biopsias } \\
\text { transbronquiales }\end{array}$ & No & $\mathrm{Si}$ & No & $\mathrm{Si}$ \\
\hline Biopsia por toracoscopia & No & No & No & No \\
\hline Coinfecciones & Citomegalovirus & No & No & Tuberculosis \\
\hline Proteína $\mathrm{C}$ reactiva & No se realizó & No se realizó & No se realizó & 12,97 \\
\hline Gases arteriales $\mathrm{PaO}_{2}$ & 54 & 66 & 73 & 58 \\
\hline Gases arteriales DA- $\mathrm{a} \mathrm{O}_{2}$ & 20 & 35 & 148 & 13 \\
\hline Gases arteriales $\mathrm{FiO}_{2}$ & $21 \%$ & $21 \%$ & $50 \%$ & $21 \%$ \\
\hline Ventilación mecánica & No & No & $\mathrm{Si}$ & No \\
\hline Muerte & No & No & $\mathrm{Si}$ & No \\
\hline Tratamiento antibiótico & $\begin{array}{l}\text { Clindamicina } \\
\text { primaquina, } 14 \text { días }\end{array}$ & TMP-SMX, 21 días & $\begin{array}{l}\text { TMP-SMX, no } \\
\text { completo }\end{array}$ & TMP-SMX, 14 días \\
\hline $\begin{array}{l}\text { Tratamiento con } \\
\text { esteroide }\end{array}$ & No & Si, 21 días & $\mathrm{Si}$, no completo & No \\
\hline
\end{tabular}


de macrófagos y la liberación de interleucina 8 y citocinas proinflamatorias $(4,7,8)$. Estos procesos promueven la fagocitosis y la destrucción del hongo, con la subsecuente liberación de proteasas y radicales libres que potencian la lesión pulmonar, reflejada en daño alveolar difuso con aparición de trastorno de la oxigenación y potencialmente falla respiratoria (9).

En una serie de casos reportada recientemente (10) la edad promedio de presentación fue de 56 años con predominio de pacientes masculinos (tasa de 0,71 hombre/mujer), siendo los diagnósticos más frecuentes el estado postransplante, las neoplasias hematológicas, las neoplasias sólidas y un caso de vasculitis. La incidencia reportada de neumonía por Pneumocystis jirovecii en pacientes inmunosuprimidos sin VIH varía de forma importante según la causa de la inmunosupresión. Dentro de los pacientes en estado postransplante, los de mayor incidencia son los cardiopulmonares con reportes de $16 \%$ a $43 \%$. En el trasplante hepático, renal y de médula ósea la incidencia es menor aunque significativa $(6,11)$. Dichas tasas han variado al compás de los cambios en los esquemas de inmunosupresión y el uso de profilaxis. De hecho, en pacientes en estado pos-trasplante la incidencia de neumonía por Pneumocystis jirovecii ha disminuido con el uso de profilaxis a valores entre el $10-15 \%$ y el $2 \%$ para hígado y riñón, respectivamente (12). Para las neoplasias hematológicas se ha descrito mayor prevalencia en algunas entidades como las leucemias agudas y los linfomas (11), especialmente asociada al uso de algunos tipos específicos de quimioterapia. Con el empleo de profilaxis la incidencia del diagnóstico en esta población ha disminuido a $0,17 \%$ en la leucemia mieloide aguda y a $0,34 \%$ en el linfoma $(6,11)$. Por otra parte, en pacientes con tumores sólidos, en especial los primarios o metastásicos a cerebro, se ha descrito una incidencia entre el 1,3 al $1,7 \%(6,13)$. Los pacientes con cáncer de pulmón o seno se consideran de riesgo elevado, en especial cuando se asocia el uso de radioterapia y esteroides (13). Dentro de las enfermedades autoinmunes, la granulomatosis de Wegener encabeza la lista con una incidencia del $6 \%(6,14)$. Incidencias mucho menores se describen en dermatomiositis, polimiositis, lupus eritematoso sistémico y artritis reumatoidea, estando estos casos descritos relacionados con el uso de corticosteriodes y terapia inmunosupresora (15). Finalmente hay reportes en la literatura de pacientes con enfermedad inflamatoria intestinal en manejo con terapia biológica.

Al analizar la casuística por medicamentos se encuentra que el uso crónico de corticosteroides, definido como periodos mayores de un mes a una dosis equivalente a más $15 \mathrm{mg}$ de prednisona, se describe como factor riesgo en $90 \%$ de los casos descritos en la literatura (16). El incremento en el uso de la llamada terapia biológica, entendida como el grupo heterogéneo de anticuerpos monoclonales y análogos de citocinas cuya utilidad ha sido demostrada en autoinmunidad, neoplasia y enfermedades inflamatorias, se ha asociado con la aparición de reportes de infección por Pneumocystis jirovecii. Un estudio reportó que tras la infusión de infliximab, la media de presentación de la neumonía por Pneumocystis jirovecii fue a las 8,5 semanas y el $76 \%$ de los pacientes había desarrollado la infección tras la semana 14 posterior a la infusión (17); no obstante, esta incidencia ha disminuido con la introducción de la profilaxis con TMP-SMX (11). Adicionalmente, se han descrito casos relacionados con el uso de los inhibidores de la calcineurina como tacrolimus y sirolimus, con bortezomib, micofenolato, ciclofosfamida, metotrexate, pemetrexed, ciclosporina, radioterapia y múltiples esquemas de antineoplásicos (18).

A diferencia del paciente con VIH donde el cuadro clínico típico está caracterizado por la presencia de síntomas respiratorios subagudos con un pronóstico relativamente benigno, la presentación en este grupo de pacientes generalmente es más aguda y severa. Los síntomas más frecuentes son: fiebre (81\%-87\% de los pacientes), tos húmeda $(71 \%-80 \%)$, disnea (66\%-68\%) y dolor torácico (23\%-24\%) (11). Los signos más comunes son: hipoxemia (78\%-91\%) y anormalidades en la auscultación pulmonar (30\%-34\%) (12). No es raro el debut con cuadro de falla respiratoria hipoxémica severa asociada a requerimiento de soporte ventilatorio (16). La mortalidad descrita es mayor a la registrada en pacientes con $\mathrm{VIH}$ y varía entre el $10 \%$ y el $60 \%$, con reportes recientes del 30 al $45 \%$ en dos grupos heterogéneos de pacientes $(19,20)$. De acuerdo con el factor riesgo, el pronóstico puede empeorar, siendo mayor la mortalidad descrita en los pacientes con cáncer (16), en los casos donde el inicio de tratamiento tarda más de siete días y ante la presencia de hipoxemia significativa y mal estado funcional de base $(19,20)$.

Existen cambios patológicos en la radiografía de tórax en 92 a $96 \%$ de los pacientes. Se describe la presencia de infiltrados intersticiales reticulares, característicamente parahiliares bilaterales, aunque también puede haber compromiso alveolar o mixto, patrones que representan el $85 \%$ de los casos (16, 21). Con frecuencia, en la tomografía de tórax se describen opacidades en vidrio esmerilado bilaterales, ya sea en parches o difusas, de predominio en lóbulos 
superiores (figuras 1, 2 y 3), así como quistes de paredes finas que se pueden asociar a neumotórax (22). También pueden ser evidentes, con menor frecuencia, la presencia de derrame pleural y linfadenopatías mediastinales (16).

Los hallazgos clínicos y radiológicos avalan la sospecha clínica y obligan a la realización de métodos invasivos con el fin de buscar la confirmación microbiológica de la infección. Teniendo en cuenta el ciclo vital y la fisiopatología del Pneumocystis jirovecii, el método utilizado más comúnmente y con muy buen rendimiento diagnóstico, sensibilidad del $77 \%$ y especificidad del $99 \%$, es el lavado broncoalveolar (BAL) por fibrobroncoscopia. Aparentemente, el esputo inducido tiene un menor rendimiento comparado con el del paciente con VIH y su uso está limitado por una disposición adecuada de la muestra y la experiencia de cada centro. Las muestras del tracto respiratorio inferior tienen la utilidad adicional de detectar posibles

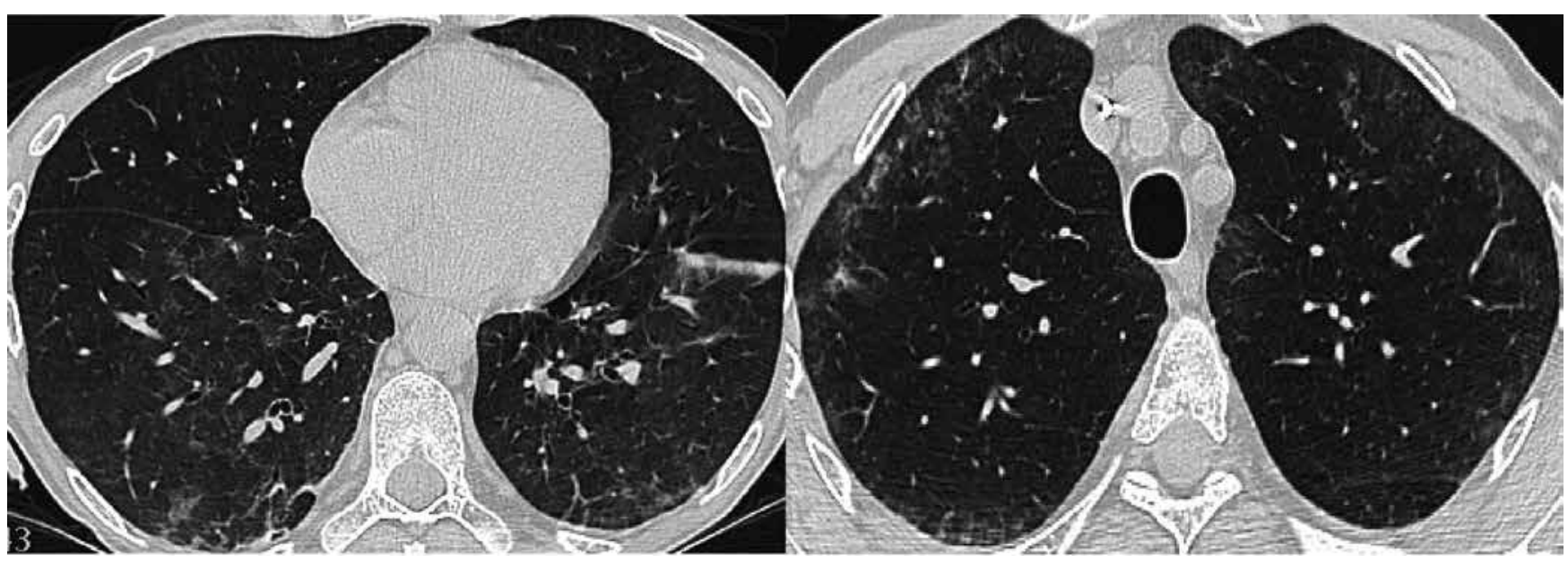

Figura 1. Paciente 1. Tomografía de tórax, ventana para pulmón: Opacidades en parche en vidrio esmerilado basal bilateral, engrosamiento intersticial interlobulillar periférico con vidrio esmerilado en lóbulos superiores.

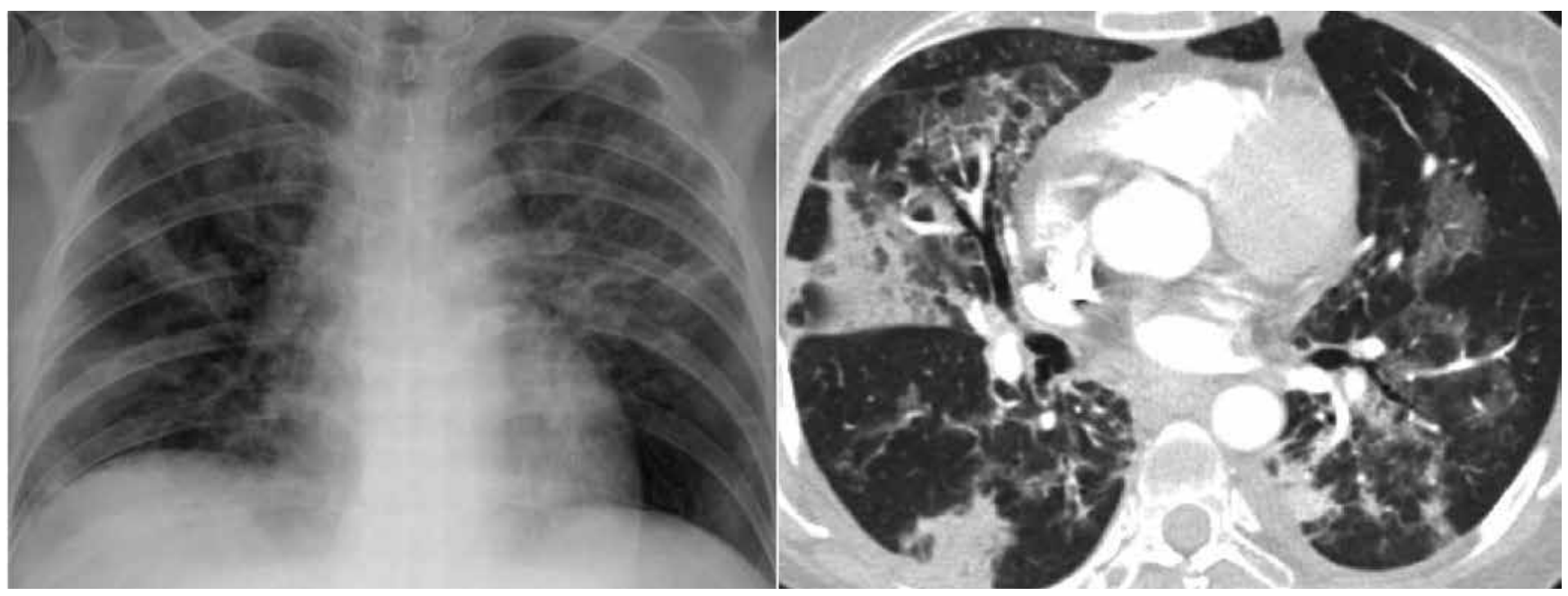

Figura 2. Paciente 2. Radiografía de tórax. Opacidades reticulares difusas predominan en los lóbulos superiores y el lóbulo inferior derecho, sin consolidación. (Izquierda). Tomografía de tórax: ventana para pulmón. Opacidades en vidrio esmerilado distribuidas en parche, patrón en empedrado en lóbulo superior derecho. 


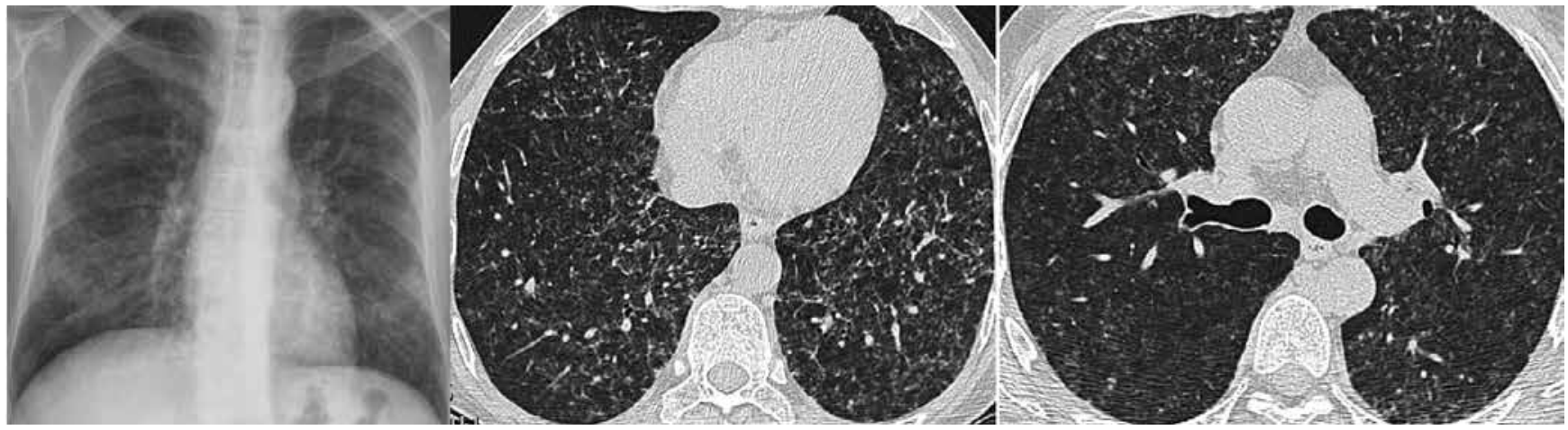

Figura 3. Paciente 3. Radiografía de tórax PA: opacidades intersticiales reticulonodulares difusas en ambos campos pulmonares. Tomografía de tórax, ventana para pulmón: micronódulos centrilobulillares, patrón en árbol de gemación de predominio en bases pulmonares, opacidades en vidrio esmerilado.

coinfecciones con otros gérmenes oportunistas, que no son infrecuentes en esta población. En dichos especímenes se pueden visualizar las dos formas de vida del Pneumocystis, trofozoitos y quistes. Con las coloraciones de hematoxilina-eosina y Papanicolaou se puede poner en evidencia la presencia de trofozoitos y su morfología es más evidente con coloraciones argénticas, como Grocott, Gomori y plata metenamina, en donde además se pueden identificar las formas quísticas (figuras 4 y 5 ). La sensibilidad y especificidad son del $49-79 \%$ y $99 \%$ respectivamente (3). A diferencia del paciente con $\mathrm{VIH}$, estos pacientes cursan con cargas bajas de microorganismos a nivel intralveolar, por lo que el

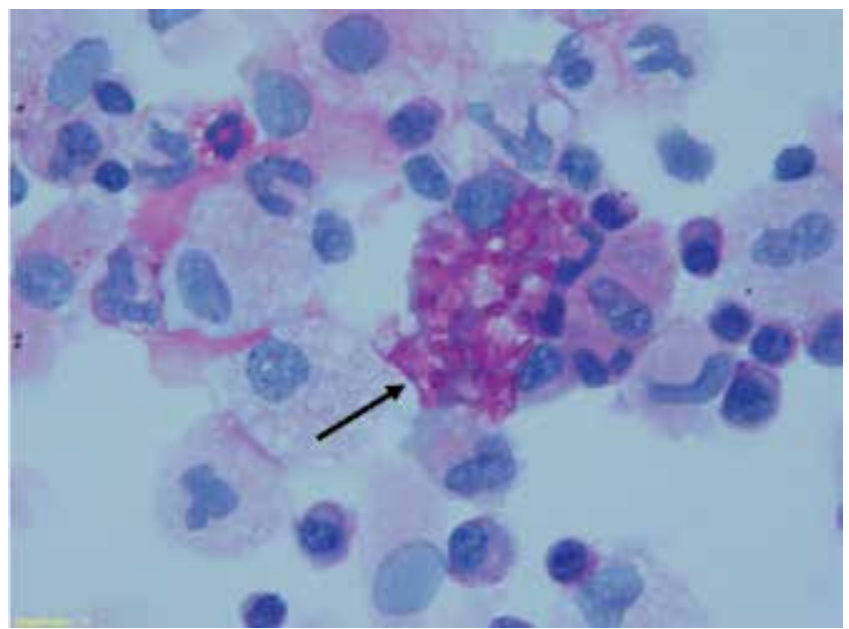

Figura 4. Lavado broncoalveolar coloración de PAS con diastasa en donde se identifican en el fondo histiocitos espumosos y células inflamatorias y los trofozoitos de P. jirovecii. Magnificación 100x. rendimiento del BAL puede verse limitado (11). Por este motivo se sugiere la toma de biopsias transbronquiales, en caso de no estar contraindicadas (figura 6). La realización de biopsia pulmonar por métodos quirúrgicos tiene un excelente rendimiento diagnóstico aunque con mayor morbimortalidad, por lo que se debe reservar para los casos excepcionales en donde los métodos broncoscópicos no son conclusivos para el diagnóstico, pero la sospecha clínica y radiológica son altas $(1,11)$.

Adicionalmente, en la literatura se describe la posibilidad de realizar estudios complementarios para la identificación del Pneumocystis jirovecii en BAL.

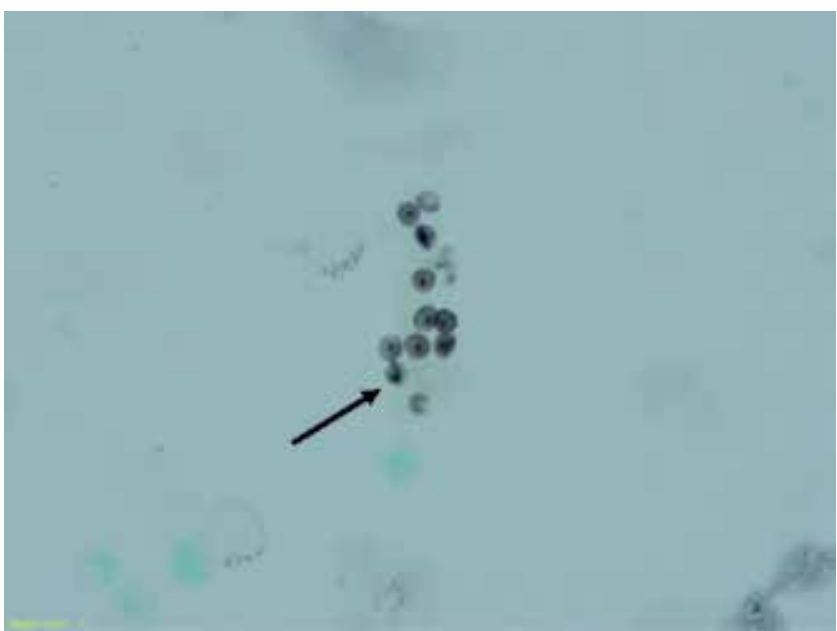

Figura 5. Lavado broncoalveolar coloración de Gomori; en un fondo limpio se identifican trofozoitos de $P$. jirovecii. Magnificación 100x. 


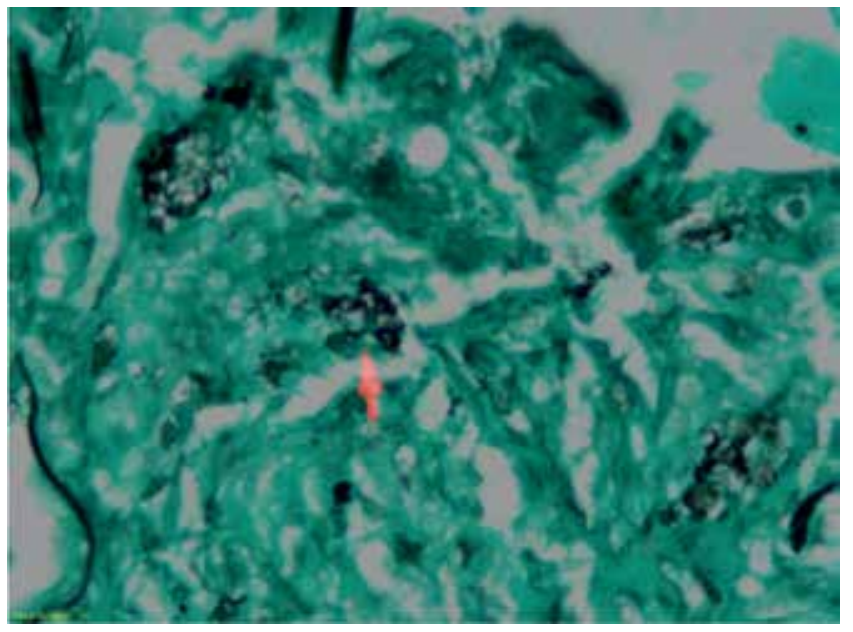

Figura 6. Biopsia transbronquial, coloración de Gomori; se identifican trofozoitos de P. jirovecii. Magnificación 100x.

Técnicas como la inmunofluorescencia de anticuerpos monoclonales, que identifica tanto trofozoitos como quistes, y la reacción de cadena de la polimerasa (PCR), que permite la detección del ADN fúngico (16), tienen excelente rendimiento, con una sensibilidad y especificidad del $91 \%$ y $95 \%$ en el caso de la primera y $94 \%$ y $96 \%$ en el caso de la segunda. Dado el riesgo de sobrediagnosticar como infección la colonización por Pneumocystis jirovecii $(16,23,24)$ en pacientes con enfermedad pulmonar obstructiva (EPOC) y otras enfermedades pulmonares crónicas, se plantea la utilización de PCR en tiempo real como alternativa para disminuir falsos positivos. La aplicabilidad de estas técnicas está limitada por su accesibilidad y costos.

Se ha planteado el uso de marcadores séricos como método alternativo de confirmación diagnóstica. Se han reportado niveles elevados de lactato deshidrogenasa (LDH) y de antígeno KL6, sintetizados por el neumocito tipo 2 y las células bronquiales; no obstante, se consideran marcadores de inflamación y de injuria inespecíficos que pueden estar elevados en procesos infecciosos pulmonares de diferentes etiologías, de ahí que su especificidad sea baja (16, 25). El 1-3 beta D-glucán es el mayor componente de la pared celular de los hongos por lo que ha sido usado para el diagnóstico de infecciones por Candida y Aspergilus (16). En el caso de la infección por Pneumocystis jirovecii, se ha propuesto su uso para diferenciar la colonización de la infección, con una sensibilidad que varía entre $88 \%-100 \%$, así como para el monitoreo a la respuesta terapéutica aunque con resultados variables $(5,11,26,27)$. Sin embargo, es preciso saber que su interpretación está limitada por la incapacidad para diferenciar entre las diferentes especies fúngicas (11) además de reportes de falsos positivos en pacientes con neumonía bacteriana o en quienes se han administrado antibióticos betalactámicos (11). Finalmente, pese a que la genotipificación ha tenido relevancia a nivel epidemiológico y microbiológico, no existe aun evidencia suficiente para promover su uso de manera indiscriminada con el objetivo de predecir desenlaces o respuesta al tratamiento.

Al igual que en los pacientes $\mathrm{VIH}$, el tratamiento de primera línea para la neumonía por Pneumocystis jirovecii en estos pacientes es el trimetoprim-sulfametoxazol (TMP-SMX) con dosis de $15-20 \mathrm{mg} / \mathrm{kg}$ para el primero y $75-100 \mathrm{mg} / \mathrm{kg}$ para el segundo $(16,11)$ (tabla 2). Dentro de los efectos adversos reportados están reacciones en piel, como necrólisis epidérmica tóxica y síndrome de Steven-Johnson, freno medular, pancreatitis, hepatitis, insuficiencia renal, hipercalemia y anafilaxia (22). La sugerencia de la duración del tratamiento de 14 ó 21 días, se definirá con base en el gradiente alveolo arterial de oxígeno. Valores mayores a $45 \mathrm{~mm} \mathrm{Hg}$ sugieren un cuadro severo, entre 35 y $45 \mathrm{~mm} \mathrm{Hg}$ uno moderado y si el gradiente es menor de $35 \mathrm{~mm} \mathrm{Hg}$ se considera cuadro leve (22). Como recomendación adicional, en el paciente no $\mathrm{VIH}$ se recomienda prolongar el tratamiento cuando hay una carga elevada del microorganismo (6).

Las opciones terapéuticas recomendadas cuando esté contraindicado el uso de sulfas son: dapsona más trimetroprim, atovaquona o clindamicina más primaquina, en casos leves y moderados, y pentamidina, en los severos (22).

El uso concomitante de corticoides en pacientes con neumonía por Pneumocystis jirovecii e infección por VIH, está avalado por estudios que demuestran disminución en la mortalidad y la morbilidad (22). Sin embargo, en los pacientes no VIH persiste como tema de controversia ya que se trata de un grupo heterogéneo en donde los resultados no son fácilmente reproducibles y los estudios no han sido concluyentes (28). Se considera, por tanto, que el uso de esteroides en este contexto debe ser individualizado y probablemente reservado para los casos severos. La dosis recomendada es de 40-60 mg de prednisona, dos veces al día por 5 a 7 días para posteriormente disminuir la dosis hasta completar 14 días (12).

Actualmente, el TMP-SMX está considerado como el medicamento de elección para la profilaxis 
Tabla 2. Tratamiento para la neumonía por Pneumocystis jirovecii.

\begin{tabular}{|c|c|c|}
\hline \multicolumn{2}{|c|}{ Medicación } & \multirow{2}{*}{$\begin{array}{l}\text { Dosis } \\
15-20 \mathrm{mg} / \mathrm{kg}+75-100 \mathrm{mg} / \mathrm{kg} \text { IV o VO; } \\
\text { dividido en } 3 \text { - } 4 \text { dosis día. }\end{array}$} \\
\hline Primera línea & Trimetroprim + Sulfametoxazol & \\
\hline \multirow{3}{*}{$\begin{array}{l}\text { Segunda línea } \\
\text { Casos leves a moderados }\end{array}$} & Dapsona + Trimetroprim & $100 \mathrm{mg} / \mathrm{d}+5 \mathrm{mg} / \mathrm{kg} 3$ veces al día. \\
\hline & Atovaquona & 750 mg 2-3 veces al día. \\
\hline & Clindamicina + Primaquina & $\begin{array}{l}600 \text { mg } 4 \text { veces al día IV } \\
350 \text { - } 400 \text { mg } 4 \text { veces al día VO + } 30 \text { mg día Vo. }\end{array}$ \\
\hline $\begin{array}{l}\text { Segunda línea } \\
\text { Casos moderados a severos }\end{array}$ & Pentamidina & 4 mg/kg día IV. \\
\hline
\end{tabular}

Tomado y modificado de: referencia 22.

IV: intravenoso; VO: vía oral.

de neumonía por Pneumocystis jirovecii (tabla 3). Esta combinación está claramente indicada durante los primeros seis meses postransplante de órgano sólido o de médula ósea (28), en especial en pacientes con coinfección por citomegalovirus, necesidad de escalamiento terapéutico inmunosupresor, uso de esteroide a más de $20 \mathrm{mg}$ de dosis equivalente de prednisona, por más de cuatro semanas, presencia de neutropenia o de actividad de autoinmunidad, trasplante pulmonar o presencia de alta incidencia de neumonía por Pneumocystis jirovecii en el centro $(>3,5 \%)$. Además, en las neoplasias hematológicas se sugiere que la profilaxis es útil sobre todo en los casos donde se requieran dosis altas de esteroides

Tabla 3. Profilaxis para Pneumocystis jirovecii.

\begin{tabular}{|lc|}
\hline \multicolumn{1}{|c|}{ Medicación } & \multicolumn{1}{c|}{ Dosis } \\
\hline TMP-SMX & Primera línea \\
& $\begin{array}{l}1 \text { tableta DS al día o } 1 \text { tableta } \\
\text { SS al día o 1 tableta DS } 3 \\
\text { veces a la semana }\end{array}$ \\
& Segunda línea \\
Dapsona & $50 \mathrm{mg} 2$ veces al día o 100 \\
& $\mathrm{mg}$ al día \\
Dapsona + pirimetamina & $50 \mathrm{mg}$ día + 50 mg semanal + \\
+ leucovorin & $25 \mathrm{mg}$ semanal \\
Dapsona + pirimetamina & $200 \mathrm{mg}$ semanal 75 mg sema- \\
+ leucovorin & nal + 25 mg semanal \\
Pentamidina & $300 \mathrm{mg}$ mensuales. Inhalado \\
Atovaquona & $1.500 \mathrm{mg}$ día \\
\hline
\end{tabular}

Tomado y modificado de: referencia 22. y durante los periodos de neutropenia (29). EI TMPSMX es el medicamento de primera línea y contribuye a prevenir otras infecciones oportunistas como el Toxoplasma gondii. En pacientes en quienes la inmunosupresión debe ser indefinida, existe controversia respecto a la continuidad de la profilaxis y se debe individualizar su uso con base en un análisis de riesgo beneficio, teniendo en cuenta que los efectos adversos del uso crónico de TMP-SMX, como son la hepatotoxicidad y la depresión medular, podrían complicar potencialmente la evolución del paciente (12). En el caso de las neoplasias hematológicas o en pacientes donde se espera recuperación del estatus inmunológico se sugiere su suspensión cuando el conteo de CD4 esté por encima de 200 (29). El uso de profilaxis en otras patologías como neoplasias sólidas, enfermedades autoinmunes o vasculitis aún se debate (tabla 4).

Cabe tener en cuenta que a pesar del uso crónico de TMP-SMX hay pacientes que pueden presentar la infección y su uso se asocia con una presentación clínica atípica que aumenta la dificultad diagnóstica al disminuir la carga fúngica, por lo que se eleva la posibilidad de requerir biopsia pulmonar para el diagnóstico.

\section{CONCLUSIÓN}

El grupo de pacientes con inmunosupresión no asociada con $\mathrm{VIH}$ tiene un riesgo variablemente aumentado de infecciones oportunistas, como se trata del caso de la neumonía por Pneumocystis jirovecii. El comportamiento clínico así como los hallazgos radiológicos, difieren al descrito típicamente en el paciente con VIH. En general, la presentación es más aguda y severa y el pronóstico más sombrío, por lo que se 
Tabla 4. Indicaciones propuestas para profilaxis.

Cáncer

- Uso de alemtuzumab durante su administración y continuar por 2 meses después del tratamiento y que CD4 > 200 cells/mL.

- Uso de temozolomida y terapia de radiación y hasta que CD4 > 200 cells $/ \mathrm{mL}$.

- Uso de fludarabina y agentes que destruyen células T (e.g., cladribine) hasta que CD4 > $200 \mathrm{cells} / \mathrm{mL}$.

- Todos los pacientes que reciben terapia antileucemia.

Enfermedades del tejido conectivo

- Granulomatosis de Wegener tratada con ciclofosfamida, especialmente si recibe corticoides.

- Vasculitis sistémica primaria tratada con corticoides o metotrexate

- Vasculitis asociada a ANCAs tratado con ciclofosfamida y corticoides.

- Artritis reumatoide tratada con inhibidores de factor de necrosis tumoral alfa y corticoides $u$ otros agentes inmunosupresores.

- Enfermedad del tejido conectivo tratada con prednisona 20 mg o más por día por más de 2 semanas.

Trasplante de células hematopoyéticas

- Receptor de trasplante alógenico por al menos 180 días.

- Receptor de trasplante autólogo de células madre de sangre periférica por 3-6 meses posterior al trasplante.

- Todos por receptores por 6 meses.

- Receptor de terapia inmunosupresora o enfermedad injerto contra hospedero por más de 6 meses.

Trasplante de órgano sólido

- Receptor de trasplante de órgano sólido por 6 a 12 meses después del trasplante.

- Receptor de trasplante renal por mínimo 4 meses después del trasplante.

- Receptor de trasplante renal por 3-6 meses después de trasplante y por lo menos 6 semanas durante y después de la terapia para evitar rechazo agudo.

Enfermedad inflamatoria intestinal

- Pacientes que reciban inhibidores de factor de necrosis tumoral alfa especialmente si usa corticoides u otro inmunosupresor.

Tomada y modificada de: referencia 3.

debe buscar de forma incisiva y rápida una confirmación microbiológica. Pese a que la carga microbiana típicamente es baja en estos pacientes, el BAL sigue siendo la herramienta fundamental para el diagnóstico, aunque se sugiere combinar con la toma de biopsias transbronquiales en ausencia de contraindicación. El uso de herramientas no invasivas, como los marcadores moleculares en el BAL o en suero, podría contribuir a un diagnóstico más certero. La toma de biopsias pulmonares quirúrgicas se restringe a los casos donde el BAL y/o las biopsias transbronquiales no corroboran la infección. Una vez confirmado el diagnóstico está indicado el inicio de TMP-SMX como medicamento de primera línea. El uso concomitante de corticoides no está indicado universalmente y se debe individualizar cada caso. En presencia de contraindicación para el uso TMP-SMX, se plantean esquemas de segunda línea de los cuales se escoge el indicado de acuerdo con la severidad del cuadro clínico. Adicionalmente, se debe realizar profilaxis de forma rutinaria en los primeros seis meses pos-trasplante. Existe controversia acerca de en que pacientes está indicada la profilaxis indefinida, especialmente en los casos en que la terapia inmunosupresora se plantee de forma permanente. Como está descrito en la literatura, los casos presentados en el artículo demuestran lo heterogéneo del grupo de pacientes inmunosuprimidos por causas diferentes al VIH y las muy importantes diferencias en la forma de presentación clínica y paraclínica de la infección pulmonar por Pneumocystis jirovecii en esta población.

\section{BIBLIOGRAFÍA}

1. Álvarez F, Bandi V, Stager C, Guntupalli KK. Detection of Pneumocystis carinii in tracheal aspirates of intubated patients using calcofluor-white (Fungi-Fluor) and immunofluorescence antibody (Genetic Systems) stains. Crit Care Med. 1997; 25: 948-952.

2. Thomas CF, Jr., Limper AH. Pneumocystis pneumonia. N Engl J Med. 2004; 350: 2487-2498.

3. Matsumura $Y$, Shindo $Y$, linuma $Y$, et al. Clinical characteristics of Pneumocystis pneumonia in non HIV patients and prognostic factors including microbiological genotypes. 
4. Fishman JA. Prevention of infection caused by Pneumocystis carinii in transplant recipients. Clin Infect Dis. 2001; 33: 1397405.

5. Damiani C, Le Gal S, Lejeune D, et al. Serum (1->3)-beta-D-glucan levels in primary infection and pulmonary colonization with Pneumocystis jirovecii. J Clin Microbiol. 2011; 49: 2000-2002.

6. Tasaka S, Tokuda H. Pneumocystis jirovecii pneumonia in nonHIV-infected patients in the era of novel immunosuppressive therapies. J Infect Chemother. 2012; 18: 793-806.

7. Limper AH, Hoyte JS, Standing JE. The role of alveolar macrophages in Pneumocystis carinii degradation and clearance from the lung. J Clin Invest. 1997; 99: 2110-2117.

8. Vassallo R, Standing JE, Limper AH. Isolated Pneumocystis carinii cell wall glucan provokes lower respiratory tract inflammatory responses. J Immunol. 2000; 164: 3755-3763.

9. Benfield TL, Vestbo J, Junge J, Nielsen TL, Jensen AB, Lundgren JD. Prognostic value of interleukin-8 in AIDS-associated Pneumocystis carinii pneumonia. Am J Respir Crit Care Med. 1995; 151: 1058-1062.

10. Toper C, Rivaud E, Daniel C, Cerf C, Parquin F, Catherinot E, et al. Pneumocystis jirovecii pneumonia in non-HIV infected patients: a study of 41 cases. Rev Pneumol Clin. 2011; 67: 191198.

11. ReidAB, ChenSC, WorthLJ. Pneumocystis jirovecii pneumonia in non-HIV infected patients: new risks and diagnostic tools. Curr Opin Infect Dis. 2011; 24: 534-44.

12. Martin SI, Fishman JA; AST Infectious Diseases Community of Practice. Pneumocystis pneumonia in solid organ transplant recipients. Am J Transplant. 2009; 9: S227-33.

13. Sepkowitz KA, Brown AE, Telzak EE, Gottlieb S, Armstrong D. Pneumocystis carinii pneumonia among patients without AIDS at a cancer hospital. JAMA. 1992; 267: 832-837.

14. Ognibene FP, Shelhamer JH, Hoffman GS, Kerr GS, Reda D, Fauci AS, et al. Pneumocystis carinii pneumonia: a major complication of immunosuppressive therapy in patients with Wegener's granulomatosis. Am J Respir Crit Care Med. 1995; 151: 795-799.

15. Ward MM, Donald F. Pneumocystis carinii pneumonia in patients with connective tissue diseases: the role of hospital experience in diagnosis and mortality. Arthritis Rheum. 1999; 42: 780-789.

16. Krajicek BJ, Thomas CF Jr, Limper AH. Pneumocystis pneumonia: current concepts in pathogenesis, diagnosis and treatment. Clin Chest Med. 2009; 30: 265-278.
17. Komano $\mathrm{Y}$, Harigai M, Koike R, et al. Pneumocystis jirovecii pneumonia in patients with rheumatoid arthritis treated with infliximab: a retrospective review and case-control study of 21 patients. Arthritis Rheum. 2009; 61: 305-312.

18. www.pneumotox.org

19. Toper C, Rivaud E, Daniel C, Cerf C, Parquin F, Catherinot E, Honderlick P, et al Pneumocystis jirovecii pneumonia in nonHIV infected patients: a study of 41 cases. Rev Pneumol Clin. 2011; 67: 191-198.

20. Ainoda Y, Hirai Y, Fujita T, Isoda N, Totsuka K; Analysis of clinical features of non HIV Pneumocystis jirovecii pneumonia. J Infect Chemother. 2012;18:722-728.

21. Drewes J, Labra W A, Tenorio J. Neumonía por Pneumocystis: reporte de un caso de evolución fulminante y actualización de su etiologia. Rev Chil Rad. 2004; 10: 172-175.

22. Catherinot E, Lanternier F, Bougnoux ME, et al. Pneumocystis jirovecii pneumonia Infect Dis Clin N Am. 2010; 24: 107-138.

23. de Oliveira A, Unnasch TR, Crothers $K$, et al. Performance of a molecular viability assay for the diagnosis of Pneumocystis pneumonia in HIV-infected patients. Diagn Microbiol Infect Dis. 2007; 57: 169-76.

24. Mühlethaler K, Bögli-Stuber K, Wasmer S, von Garnier C, Dumont P, Rauch A, et al. Quantitative PCR to diagnose Pneumocystis pneumonia in immunocompromised non-HIV patients. Eur Respir J. 2012; 39: 971-8.

25. Larsen $\mathrm{HH}$, Huang $\mathrm{L}$, Kovacs JA, et al. A prospective, blinded study of quantitative touch-down polymerase chain reaction using oral-wash samples for diagnosis of Pneumocystis pneumonia in HIV infected patients. J Infect Dis. 2004; 189: 1679-83.

26. Held J, Koch MS, Reischl U, et al. Serum (1 -> 3)-beta-D-glucan measurement as an early indicator of Pneumocystis jirovecii pneumonia and evaluation of its prognostic value. Clin Microbiol Infect. 2011; 17: 595-602.

27. Marty FM, Koo S, Bryar J, Baden LR. (1->3)beta-D-Glucan assay positivity in patients with Pneumocystis (carinii) jirovecii pneumonia. Ann Intern Med. 2007; 147: 70-72.

28. Moon SM, Kim T, Sung $H$, et al. Outcomes of moderate to severe Pneumocystis pneumonia treated with adjunctive steroid in nonHIV-infected patients. Antimicrob Agents Chemother. 2011; 55: 4613-8.

29. De Castro N, Pavie J, Lagrange-Xélot M, Molina JM. Pneumocystose chez les patients d'onco-hématologie : est-ce inévitable ?; Revue des Maladies Respiratoires. 2007; 24: 741-750. 